\title{
Improving SVM Classification on Imbalanced Datasets by Introducing a New Bias
}

\author{
Haydemar Núñez \\ Central University of Venezuela, Venezuela \\ Luis Gonzalez-Abril \\ Universidad de Sevilla, España \\ Cecilio Angulo \\ Technical University of Catalonia, Spain
}

\begin{abstract}
Support Vector Machine (SVM) learning from imbalanced datasets, as well as most learning machines, can show poor performance on the minority class because SVMs were designed to induce a model based on the overall error. To improve their performance in these kind of problems, a low-cost post-processing strategy is proposed based on calculating a new bias to adjust the function learned by the SVM.

The proposed bias will consider the proportional size between classes in order to improve performance on the minority class. This solution avoids not only introducing and tuning new parameters, but also modifying the standard optimization problem for SVM training.

Experimental results on 34 datasets, with different degrees of imbalance, show that the proposed method actually improves the classification on imbalanced datasets, by using standardized error measures based on sensitivity and g-means. Furthermore, its performance is comparable to well-known cost-sensitive and Synthetic Minority Over-sampling Technique (SMOTE) schemes, without adding complexity or computational costs.
\end{abstract}

Keywords: Support Vector Machine; Post-processing; Bias; Cost-sensitive strategy: SMOTE.

This research is partially supported by the project of the Spanish Ministry of Economy and Competitiveness, HERMES (TIN2013-46801-C4-1-R) and Simon (TIC-8052) of the Andalusian Regional Ministry of Economy, Innovation and Science.

Corresponding Author's Address: H. Núñez, Escuela de Computación, Facultad de Ciencias, Universidad Central de Venezuela, Paseo Los Ilustres Caracas 1040, Venezuela, email: haydemar.nunez@ciens.ucv.ve.

Published online: 14 October 2017 


\section{Introduction}

A major problem faced by classification learning algorithms is the imbalance between classes in datasets. It appears when there are many examples of one or several classes, but very few in the remaining classes. Some domains where this situation arises are medical diagnosis, text classification, fraud detection in credit card usage, detection of communication network intrusion, among others. Since it usually represents the target of the classification task, for such scenarios is very important to obtain models that exhibit a high prediction performance on the minority class. However, standard learning algorithms tend to exhibit good performance only on the majority class, because they construct classification models based on error over the whole training set, independently of the representatives or balance between classes.

To solve this problem, some mechanisms exist to allow these algorithms to show good performance on minority class. To that effect, several strategies have been proposed, such as re-balancing the dataset with sampling techniques, construction of classifiers that take into account the cost of errors on different classes, and combination (ensemble) of results from several classifiers trained with different data distributions (He and Garcia, 2009; López et al., 2013; Sun, Wong, and Kamel, 2009).

In the case of SVMs, their learning mechanism becomes an interesting option to deal with imbalanced datasets, because SVMs build their classification model based only on a subset of training instances (Cristianini and Shawe-Taylor, 2000; Vapnik, 1999). However, like other machine learning techniques, SVMs minimize the error over all the dataset to generate these models, so they are biased towards the majority class when the imbalance is severe.

To enhance the performance of SVMs for problems with imbalanced classes, several solutions have been proposed. Some of them are of general application, like sampling techniques to re-balancing datasets in a preprocessing stage; other, more specific, consider SVM's particular features like those based on cost-sensitive learning (Batuwita and Palade, 2013). Some research papers suggest using a post-processing stage to reduce the bias towards the majority class of the classifier learned by the SVM (He and Garcia, 2009).

Following this last research line, a strategy for SVMs based on calculating a new bias or threshold is proposed. This new bias considers the classes' proportion in the dataset and allows tuning the original function learned by the SVM to improve its performance on the minority class. The proposed solution neither introduces new parameters, nor modifies the original optimization problem for SVM training. 
This paper is organized as follows: Section 2, briefly introduces the SVM learning mechanism and provides an overview of strategies to improve its performance on these kind of problems. In Section 3, the proposed post-processing procedure for determining the new bias is detailed. Section 4, presents the experiments performed to verify the applicability of the proposal, along with an analysis of results, and a comparison between performance of the new approach and a cost-sensitive scheme. Finally, conclusions and further research are presented.

\section{SVM on Imbalanced Datasets}

SVMs are based on statistical learning theory and have been applied successfully in classification and regression problems in different domains (Cristianini and Shawe-Taylor, 2000; Oneto, Ridella, and Anguita, 2016; Vapnik, 1999). The hypothesis space of these learning machines are hyperplanes (linear decision surfaces). Training looks for a decision function with the maximum margin of separation between classes. Thus, for a binary classification task on a set of training data $\mathcal{Z}=\left\{\left(\mathbf{x}_{1}, y_{1}\right), \ldots,\left(\mathbf{x}_{N}, y_{N}\right)\right\}$, with $\mathbf{x}_{i} \in \mathcal{X} \subseteq \Re^{m}, y_{i} \in \mathcal{Y}=\{+1,-1\}$, and the decision function $f(x)=\mathbf{w} \cdot \mathbf{x}-b$, the optimal hyperplane is determined as follows,

$$
\begin{aligned}
& \min _{\mathbf{w}, b} \frac{1}{2}\|\mathbf{w}\|^{2}+C \sum_{i=1}^{N} \xi_{i} \\
& \text { s. t. }\left\{\begin{array}{l}
y_{i}\left(\mathbf{w} \cdot \mathbf{x}_{i}-b\right)+\xi_{i} \geq 1, \quad i=1 \ldots N \\
\xi_{i} \geq 0,
\end{array}\right.
\end{aligned}
$$

where $\mathrm{w}$ is the vector of the hyperplane which defines its orientation, and $b$ is the bias which determines its position. Slack variables, $\xi_{i}$, measure the error on the instances that violate the constraint $y_{i}\left(\mathbf{w} \cdot \mathbf{x}_{i}-b\right) \geq 1$. The user-defined parameter, $C$, determines the trade-off between maximizing the margin and minimizing the error, i.e. for higher values of $C$, the SVM focuses more on minimizing errors. In a dual form, this optimization problem can be solved as,

$$
\begin{aligned}
\max _{\alpha_{i} \in \Re} & \sum_{i=1}^{N} \alpha_{i}-\frac{1}{2} \sum_{i, k=1}^{N} \alpha_{i} y_{i} \alpha_{k} y_{k} \mathbf{x}_{i} \cdot \mathbf{x}_{k} \\
\text { s.t. } & \left\{\begin{array}{l}
0 \leq \alpha_{i} \leq C, \quad i=1 \ldots N \\
\sum_{i=1}^{N} \alpha_{i} y_{i}=0,
\end{array}\right.
\end{aligned}
$$

leading to the following decision function, 


$$
f(\mathbf{x})=\operatorname{sign}\left(\sum_{i=1}^{N} \alpha_{i} y_{i} \mathbf{x}_{i} \cdot \mathbf{x}-b\right) .
$$

To construct nonlinear decision boundaries, input vectors are projected in an inner product space of higher dimension using a basis set of nonlinear functions. In this new space, the optimal hyperplane is determined. Using the theory of kernels satisfying Mercer's theorem, all operations can be performed directly in an input space using $\mathbf{x}_{i} \cdot \mathbf{x}_{j}=K\left(\mathbf{x}_{i}, \mathbf{x}_{j}\right)$. Then, the decision function is formulated as,

$$
f(\mathbf{x})=\operatorname{sign}\left(\sum_{i=1}^{N} \alpha_{i} y_{i} K\left(\mathbf{x}_{i}, \mathbf{x}_{j}\right)-b\right) .
$$

Among all the training vectors, only a few have an associated weight $\alpha_{i}$ greater than zero in (3) or (4). These elements lie in the decision margin and are known as support vectors (SV). The unsigned value $f(\mathbf{x})$ is a measure of the distance of an example $\mathbf{x}$ to the hyperplane, while the sign determines the class label (positive or negative).

For moderately imbalanced datasets, empirical results show that, unlike other machine learning algorithms, SVMs can produce a good hypothesis without any modification (Akbani, Kwek, and Japkowicz, 2004; Imam, Ting, and Kamruzzaman, 2006; Wu and Chang, 2005). One explanation for such phenomenon is that SVMs use only a set of support vectors to construct classification models, so negative instances that are far from the decision border will not be taken into account and the SVM will not be affected by them, even the numerous ones. However, SVMs cannot overcome the problem of imbalance when the data distribution is very imbalanced. In such cases, it has been observed that the hyperplane separation learned by the SVM is very close to the minority class, resulting in a low performance or no generalization at all for examples from this class, in comparison with those from the majority class (Batuwita and Palade, 2013; He and Ghodsi, 2010; Liu, An, and Huang, 2006, Wu and Chang, 2005).

\subsection{Strategies for SVM with Imbalanced Datasets}

Several strategies have been proposed to improve the performance of SVMs on imbalanced datasets. Some of them are described and introduced in this section according to the moment that they can be applied during the learning process. 


\subsubsection{Pre-Processing Strategies}

They are based on re-sampling techniques to balance the dataset. One way is through the over-sampling of data from the minority class; hence, new instances are created to increase its proportion in the dataset. In contrast, under-sampling seeks to reduce the size of the majority class by removing a subset of these data. They are general-purpose procedures, not targeted at any particular machine learning techniques. One of the most commonly used is Synthetic Minority Over-sampling Technique (SMOTE), which employs the $k$-nearest neighbor technique for over-sampling the minority class (Chawla et al., 2002; Vilariño et al., 2005). Others strategies apply clustering algorithms for sub-sampling the majority class ( $\mathrm{Li}$ et al., 2014; Yu et al., 2006; Zhou, Ha, and Wang, 2010).

There are also strategies for the SVM that seeks increasing the minority class by considering the margin area between the two classes (Castro, Carvalho, and Braga, 2009). Other works are based on the use of SVM to obtain the positive support vectors, and over-sample from these data (Hernández-Santiago et al., 2012; Wang, 2008). This feature has also been exploited to build under-sampling algorithms (Tang et al., 2009; Wang, 2014), where an SVM is used to build a new dataset composed only of the most informative negative support vectors and the positive data. Other solutions use sampling methods with ensembles (Kang and Cho, 2006; Liu et al., 2006; Waske, Benediktsson, and Sveinsso, 2009; Yang et al., 2011; Sukhanov et al., 2015). Furthermore, there are proposals that seek oversampling during training by using active learning (Ertekin, 2013).

\subsubsection{Training Strategies}

These strategies include those proposals that modify the standard optimization problem for SVM training to incorporate information related to the proportion of classes in the dataset. One approach of cost-sensitive learning incorporates into the learning problem information related with the penalties associated with wrong predictions for each class. In the case of SVMs, the cost information about the two types of errors can be introduced into the formulation of the learning problem, using two regularization parameters, $C^{+}$and $C^{-}$, associated with errors on the positive and negative class, respectively (Veropoulos, Campbell, and Cristianini, 1999; Cohen et al., 2006),

$$
\begin{aligned}
& \min _{\mathbf{w}, b} \frac{1}{2}\|\mathbf{w}\|^{2}+C^{+} \sum_{i \mid y_{i}=+1} \xi_{i}+C^{-} \sum_{i \mid y_{i}=-1} \xi_{i} \\
& \text { s.t. }\left\{\begin{array}{l}
y_{i}\left(\mathbf{w} \cdot \mathbf{x}_{i}-b\right)+\xi_{i} \geq 1, \quad i=1 \ldots N . \\
\xi_{i} \geq 0,
\end{array}\right.
\end{aligned}
$$


Some works have also added new restrictions on the slack variables, $\xi_{i}$, to control the margin of separation between the two classes (He and Ghodsi, 2010; Yang et al., 2008). A different approach is presented in Batuwita and Palade (2010), where only one regularization parameter, $C$, is used, but information about the cost of errors is incorporated by allocating different weights to each variable $\xi_{i}$. Other proposed solutions combine cost-sensitive learning with other techniques (Akbani et al., 2004; Muscat et al., 2014; Wang and Japkowicz, 2010; Zięba et al., 2014).

Other proposals are those that modify the kernel matrix according to the observed imbalance in the distribution of data, such as the KBA algorithm (Wu and Chang, 2005). In Ramírez and Allende (2012), a method is proposed such that training two one-class SVMs, one fitted to each class, and aggregating their decisions in a nested manner, the boundary is improved. Finally, in He et al. (2015), a model-based approach integrating cost-sensitive learning with Gaussian Mixture Models for the imbalanced classification problem is proposed.

\subsubsection{Post-Processing Strategies}

In general, these approaches are oriented either, towards modifying the weight vector $\mathbf{w}$ in the function of decision or determining a new bias, in order to adjust the decision boundary learned by the SVM so as to provide a good margin of separation for the positive class. For example, the $z$-SVM method is proposed in Imam et al. (2006), which determines the value of a new parameter, $z$, solving an added optimization problem. This optimal parameter weights the contribution of support vectors of the minority class in the vector $\mathbf{w}$ of the decision function, obtained after training.

In $\mathrm{Li}, \mathrm{Hu}$, and Hirasawa (2008), the bias of the decision function is modified by calculating an offset $\theta$ from the average of the unsigned values generated by $f(\mathbf{x})$ for the support vectors. A similar strategy is used in Shanahan and Roma (2003), with the new offset being calculated by applying the Beta-Gamma algorithm.

Other studies suggest re-interpreting the outputs of the SVM. For example, a fuzzy decision function is applied in Li et al. (2008), whose parameters are estimated from the observed distribution in the dataset. In Wang and Zheng (2008), the decision process incorporates a post-processing module, whose construction is based on methods of information theory to define a new bias for classification.

\section{A Novel Post-processing Strategy Based on the Bias}

Strategies proposed to improve the performance of SVM on imbalanced datasets generally require tuning new parameters such as the sample 
rate or the number $k$ of selected neighbors. Other methods can be computationally expensive considering the construction of several classifiers (such as methods based on ensembles), or based on iterative algorithms, such as modifying the kernel matrix (KBA), and some sampling techniques that require several over-training steps. In cost-sensitive approaches, the standard SVM optimization problem must be modified and costs of errors on the classes must be known. Moreover, they can produce over-fitted models (Wang and Japkowicz, 2010).

On the other hand, it has been empirically shown that the hyperplane learned by SVMs in presence of imbalanced datasets have approximately the same orientation as the ideal hyperplane (He and Ghodsi, 2010; Liu et al., 2006; $\mathrm{Wu}$ and Chang, 2005). Reduced generalization on the minority class would be indeed associated with the bias $b$, as positive instances lie far from this ideal limit, i.e., the SVM learns a boundary that is too close to this class. Other studies, such as those presented in Sun, Lim, and Liu (2009) in the domain of text classification, suggest increasing research on strategies determining new thresholds for the SVM's decision function. Modifications should be based on the distribution of classes in the dataset, which also do not directly affect standard SVM training.

Following the latter research line, a novel post-processing strategy based on calculating a new bias is proposed in this paper. The proportion among classes in the dataset will be considered, hence adjusting the function learned by the SVM to improve their performance on the minority class. The proposed solution does not involve tuning new parameters. Furthermore, it neither requires modifying the standard optimization problem for training the SVM, nor additional steps of re-training.

The proposal, based on the developments presented in Gonzalez-Abril et al. (2008), modifies, after training, the separating margin of the hyperplane towards the majority class to achieve better generalization performance on data from the minority class. The new bias is calculated as follows (Núñez, Gonzalez-Abril, and Angulo, 2011).

Let $\mathcal{Z}=\left\{\left(\mathbf{x}_{1}, y_{1}\right), \ldots,\left(\mathbf{x}_{N}, y_{N}\right)\right\}$ be a training set, with $\mathbf{x}_{i} \in \mathcal{X} \subseteq$ $\Re^{m}, y_{i} \in \mathcal{Y}=\{+1,-1\}$. Also, let $\mathcal{Z}_{1}$ and $\mathcal{Z}_{2}$ be the datasets belonging to the positive class (+) and the negative one (-), respectively. The standard formulation of the bias, for the linearly separable case indicates that bias could be obtained as,

$$
b_{s}=\frac{\alpha+\beta}{2}
$$

$\left(b_{s}=b_{\text {standard }}\right)$ where $\alpha$ is the maximum value of the hyperplane without bias applied to the set of negative instances $\mathcal{Z}_{2}$, and $\beta$ is the minimum value of the hyperplane without bias applied to the entries in the minority set $\mathcal{Z}_{1}$, 
that is,

$$
\alpha=\max _{\mathbf{x}_{k} \in \mathcal{Z}_{2}} \sum_{i=1}^{N} \alpha_{i} K\left(\mathbf{x}_{i}, \mathbf{x}_{k}\right), \quad \text { and } \quad \beta=\min _{\mathbf{x}_{k} \in \mathcal{Z}_{1}} \sum_{i=1}^{N} \alpha_{i} K\left(\mathbf{x}_{i}, \mathbf{x}_{k}\right) .
$$

Let us indicate that if the bias $b$ is chosen as $b=\beta$, then all instance of the positive class are correctly labeled. Furthermore, $\beta$ is the smallest value that ensures a $100 \%$ correct classification of the training vectors (GonzalezAbril et al., 2014).

Definition for $b_{s}$ in (6) has been extended to take into account the proportion of classes in the dataset. Hence, for $N_{1}$ and $N_{2}$ being the number of patterns in classes (+) and (-), respectively, a new proportional bias $b_{p}$ is defined,

$$
b_{p}=\frac{N_{1} \alpha+N_{2} \beta}{N_{1}+N_{2}} .
$$

Hence, for imbalanced problems, $N_{1} \ll N_{2}$, this new bias will move the decision limit towards the negative class, thus increasing the margin of separation for the positive class. Moreover, as the maximum and minimum values for the hyperplane without bias are reached on support vectors, only these points need to be considered for calculation of $\alpha$ and $\beta$,

$$
\alpha=\max _{\mathbf{x}_{k} \in S V_{2}} \sum_{i=1}^{N} \alpha_{i} K\left(\mathbf{x}_{i}, \mathbf{x}_{k}\right), \quad \text { and } \quad \beta=\min _{\mathbf{x}_{k} \in S V_{1}} \sum_{i=1}^{N} \alpha_{i} K\left(\mathbf{x}_{i}, \mathbf{x}_{k}\right)
$$

where $S V_{1}$ and $S V_{2}$ are the set of support vector in classes (+) and (-), respectively. The new decision function would simply be expressed as follows,

$$
f(\mathbf{x})=\operatorname{sign}\left(\sum_{i=1}^{N} \alpha_{i} y_{i} K\left(\mathbf{x}_{i}, \mathbf{x}_{j}\right)-b\right) .
$$

Furthermore, as the SVM decision function is generated only from the support vectors (the most informative instances for the classification task), an additional modification to this proposal exists: to consider the number of support vectors for the positive and negative classes, $N_{s v 1}$ and $N_{s v 2}$, respectively, rather than values $N_{1}$ and $N_{2}$.

Hence, a second bias $b_{p 1}$ is also proposed,

$$
b_{p 1}=\frac{N_{s v 1} \alpha+N_{s v 2} \beta}{N_{s v 1}+N_{s v 2}} .
$$

Let us see the relationship among these biases for $N_{1} \ll N_{2}$. In this case, the optimization problem usually provides a number of support vectors 
such that $N_{s v 1}<N_{s v 2}$ and, defining $R=N_{2} / N_{1}$ and $r=N_{s v 2} / N_{s v 1}$, then $1 \leq r \leq R$ (this fact can be checked in Section 4). Hence, it results,

$$
\begin{aligned}
\left|\beta-b_{p}\right| & =\frac{1}{1+R}|\beta-\alpha|, \\
\left|\beta-b_{p 1}\right| & =\frac{1}{1+r}|\beta-\alpha|, \\
\left|\beta-b_{s}\right| & =\frac{1}{2}|\beta-\alpha|,
\end{aligned}
$$

and

$$
0 \leq\left|\beta-b_{p}\right| \leq\left|\beta-b_{p 1}\right| \leq\left|\beta-b_{s}\right|
$$

that is, $b_{s}$ is farther from $\beta$ than $b_{p 1}$, that in its turn, is farther away from $\beta$ than $b_{p}$. Thus, it can be checked that the decision function is moving away from the zone of the positive samples, increasing, as it will be later demonstrated, the accuracy on this class. Therefore, these new biases move the hyperplane learned by SVM to obtain a better classification performance for the positive class, considering the proportions of the classes: the greater the imbalance, the greater margin of separation for the minority class.

\section{Experimentation and Results Analysis}

Performance of post-processing strategy proposed was tested on 34 datasets from the UCI repository (Frank and Asuncion 2010). Characteristics of the datasets are shown in Table 1. Label (+) was assigned to the class shown in brackets, and label (-) to the remaining data. The performance of the classifiers obtained by using the new biases was measured using sensitivity and geometric mean (g-means) (He and Garcia, 2009). Sensitivity measures positive accuracy, indicating how many examples of the minority classes are correctly classified; g-means evaluates the performance in terms of sensitivity and specificity (negative accuracy) as follows,

$$
\text { g-means }=\sqrt{\text { sensitivity } \cdot \text { specificity }}
$$

Sensitivity allows us to show how well the positive class is classified and g-means shows the balance between the accuracy of positive and negative classes. Also, accuracy was included, as being the standard metric. For SVM training, the usual RBF kernel was used, as well as the Matlab's Bioinformatics Toolbox for processing. The values of $\sigma$ (RBF width) and $C$ (regularization term) were obtained by exploring a two-dimensional grid: 
Table 1. UCI datasets used in the experimentation. These datasets are ordered from extreme to moderate imbalance.

\begin{tabular}{|c|c|c|c|c|c|c|c|}
\hline Datasets & $\begin{array}{r}\text { numb } \\
\text { instances }\end{array}$ & $\begin{array}{l}\text { er of } \\
\text { positives }\end{array}$ & $\begin{array}{l}6 \text { of } \\
\text { ositives }\end{array}$ & Datasets & $\begin{array}{r}\text { num } \\
\text { nstances }\end{array}$ & $\begin{array}{l}\text { ber of } \\
\text { positives }\end{array}$ & $\begin{array}{l}\% \text { of } \\
\text { ositives }\end{array}$ \\
\hline Winequality_white (3) & 3) 4868 & 20 & 0.41 & User Modeling (1) & 258 & 24 & 9.30 \\
\hline Abalone (19) & 4177 & 32 & 0.77 & Sat (4) & 4435 & 415 & 9.36 \\
\hline Winequality_red (8) & 1593 & 18 & 1.18 & Satimage (4) & 6435 & 626 & 9.70 \\
\hline Page-Blocks (5) & 5473 & 115 & 2.10 & Euthyroid & 2000 & 238 & 11.90 \\
\hline Yeast (7) & 1483 & 35 & 2.36 & Glass (7) & 214 & 29 & 13.55 \\
\hline Thyroid (1) & 3772 & 93 & 2.47 & Segment (1) & 2310 & 330 & 14.29 \\
\hline Nursey (3) & 12960 & 328 & 2.53 & Hepatitis & 129 & 24 & 18.60 \\
\hline Fault (5) & 1941 & 55 & 2.83 & Column & 310 & 60 & 19.35 \\
\hline Winequality_white (4) & 4864 & 163 & 3.35 & $\mathrm{Cmc}(2)$ & 1473 & 333 & 22.61 \\
\hline Yeast (5) & 1483 & 51 & 3.44 & Dna & 2000 & 464 & 23.20 \\
\hline Muns (3) & 8124 & 292 & 3.59 & Vehicle (1) & 846 & 199 & 23.52 \\
\hline Letter (a) & 20000 & 789 & 3.95 & Transfusion & 748 & 178 & 23.80 \\
\hline Car (3) & 1728 & 69 & 3.99 & Haberman & 306 & 81 & 26.50 \\
\hline Derma (2) & 358 & 21 & 5.87 & German & 1000 & 300 & 30.00 \\
\hline Ecoli (5) & 336 & 20 & 5.95 & Waveform (0) & 5000 & 1657 & 33.00 \\
\hline Balance(2) & 625 & 49 & 7.24 & Pima & 768 & 268 & 34.00 \\
\hline GTC & 2126 & 176 & 8.28 & TicTac (2) & 958 & 332 & 34.66 \\
\hline
\end{tabular}

$\sigma=\left\{2^{0}, 2^{1}, \ldots, 2^{6}\right\}, C=\left\{2^{0}, 2^{1}, \ldots, 2^{10}\right\}$ and the best values for accuracy for each classifier (SVM, cost-sensitive SVM and SMOTE SVM) were used.

Average values for accuracy, g-means and sensitivity are shown in Table 2, for each dataset, using ten-fold cross-validation like empirical experimentation and repeating this procedure 10 times to ensure a good statistical behavior. From these results some statements can be established:

- Working with imbalanced datasets, evaluation metrics like g-means and sensitivity measure the classifiers performance independently of the data distribution, so their election is correct for this kind of problems. For example, SVM has an accuracy value of 0.99 over Abalone dataset. However, it completely fails classifying the positive class, which is reflected in the value of g-means.

- For some datasets, despite the imbalance, the original SVM can get a reasonable model (e.g. Ecoli); but in other cases, it fails (Abalone, Winequality, Yeast).

- The new biases improve the performance of the standard SVM in all datasets with respect to g-means and sensitivity metrics. Furthermore, the performance on the sensitivity metric when using the $b_{p}$ bias is better than employing $b_{p 1}$, in all datasets except for Tic-Tac dataset. 
Table 2. Average values for accuracy, g-means and sensitivity for each dataset, using ten-fold cross-validation (100 replications).

\begin{tabular}{|c|c|c|c|c|c|c|c|c|c|}
\hline \multirow[t]{2}{*}{ Datasets } & \multicolumn{3}{|c|}{ accuracy } & \multicolumn{3}{|c|}{ g-means } & \multicolumn{3}{|c|}{ sensitivity } \\
\hline & $\overline{\mathrm{SVM}}$ & $b_{p}$ & $b_{p 1}$ & SVM & $b_{p}$ & $b_{p 1}$ & SVM & $b_{p}$ & $b_{p 1}$ \\
\hline Wineq_white (3) & .996 & .968 & .969 & .120 & .449 & .449 & .038 & .235 & .235 \\
\hline Abalone (19) & .992 & .491 & .619 & .000 & .625 & .552 & .000 & .828 & .592 \\
\hline Wineq_red (8) & .989 & .506 & .813 & .000 & .603 & .296 & .000 & .835 & .280 \\
\hline Page-Blocks (5) & .984 & .761 & .892 & .534 & .858 & .803 & .296 & .974 & .821 \\
\hline Yeast (7) & .978 & .649 & .961 & .300 & .749 & .831 & .143 & .891 & .723 \\
\hline Thyroid (1) & .992 & .928 & .992 & .851 & .95 & .886 & .733 & .976 & .794 \\
\hline Nursey (3) & .993 & .981 & .984 & .878 & .983 & .602 & .774 & .986 & .37 \\
\hline Fault (5) & .955 & .938 & .951 & .323 & .594 & .432 & .166 & .417 & .247 \\
\hline Wineq_white 4) & .963 & .764 & .921 & .336 & .621 & .568 & .128 & .513 & .353 \\
\hline Yeast (5) & .965 & .464 & .953 & .000 & .628 & .623 & .000 & .907 & .453 \\
\hline Muns (3) & .954 & .953 & .954 & .572 & .903 & .587 & .349 & .858 & .366 \\
\hline Letter (a) & .998 & .994 & .998 & .975 & .995 & .994 & .951 & .996 & .991 \\
\hline Car (3) & .960 & .973 & .968 & .000 & .941 & .595 & .000 & .911 & .39 \\
\hline Derma (2) & .968 & .951 & .957 & .923 & .944 & .935 & .864 & .936 & .906 \\
\hline Ecoli (5) & .986 & .968 & .974 & .851 & .950 & .902 & .775 & .945 & .855 \\
\hline Balance(2) & .921 & .774 & .845 & .749 & .830 & .851 & .623 & .909 & .867 \\
\hline GTC & .980 & .882 & .975 & .912 & .920 & .950 & .804 & .970 & .924 \\
\hline User modeling (1) & .979 & .981 & .985 & .885 & .968 & .971 & .791 & .956 & .956 \\
\hline Sat (4) & .948 & .868 & .943 & .787 & .894 & .837 & .634 & .929 & .727 \\
\hline Satimage (4) & .945 & .911 & .942 & .811 & .889 & .83 & .678 & .865 & .716 \\
\hline Euthyroid & .907 & .761 & .889 & .703 & .809 & .809 & .517 & .887 & .727 \\
\hline Glass (7) & .951 & .879 & .953 & .855 & .898 & .868 & .768 & .935 & .792 \\
\hline Segment (1) & .996 & .993 & .996 & .988 & .994 & .991 & .977 & .995 & .984 \\
\hline Hepatitis & .852 & .721 & .741 & .643 & .732 & .744 & .562 & .855 & .84 \\
\hline Column & .867 & .872 & .868 & .758 & .873 & .714 & .645 & .883 & .57 \\
\hline $\mathrm{Cmc}(2)$ & .759 & .630 & .714 & .527 & .618 & .594 & .320 & .607 & .452 \\
\hline Dna & .967 & .949 & .957 & .948 & .957 & .96 & .914 & .972 & .967 \\
\hline Vehicle (1) & .986 & .983 & .985 & .982 & .985 & .985 & .975 & .988 & .986 \\
\hline Transfusion & .779 & .748 & .778 & .540 & .614 & .557 & .327 & .462 & .350 \\
\hline Haberman & .719 & .634 & .661 & .464 & .617 & .609 & .273 & .606 & .512 \\
\hline German & .762 & .623 & .708 & .667 & .660 & .695 & .517 & .804 & .680 \\
\hline Waveform (0) & .897 & .873 & .895 & .877 & .884 & .863 & .824 & .921 & .783 \\
\hline Pima Diabetes & .755 & .742 & .753 & .672 & .725 & .676 & .518 & .687 & .530 \\
\hline TicTac (2) & .983 & .997 & .997 & .974 & .996 & .997 & .950 & .995 & .998 \\
\hline
\end{tabular}

This fact is due to that the Tic-Tac dataset is the unique of the 34 datasets such that $r \leq R$ is not true.

To compare the performance of this post-processing strategy with other reported in the literature, both, SMOTE and a cost-sensitive scheme were used to train a SVM on the listed UCI datasets. Comparison was only made with the $b_{p}$ bias. The Matlab Bioinformatic's toolbox provides a cost- 
sensitive scheme where the values for $C^{+}$and $C^{-}$in (5) are calculated from $C$ as:

$$
C^{+}=C \frac{N}{2 N_{1}}, \quad \text { and } \quad C^{-}=C \frac{N}{2 N_{2}} .
$$

It is worth noting that from the above, $C=\frac{C^{+} N_{1}+C^{-} N_{2}}{N_{1}+N_{2}}$, that is, a similar formula to the bias $b_{p}$ by changing $C^{+}$and $C^{-}$for $\alpha$ and $\beta$, respectively.

Results obtained using the same evaluation metrics, as well as the same ten-fold cross-validation structure, are shown in Table 3. Moreover, a comparison about the proportion of support vectors of the learned decision function in relation with the number of training data is offered for all the schemes in Table 4. This ratio is a measure of the complexity of the SVM classifier. Therefore, it can be concluded than both, SMOTE and cost sensitive approaches provide a decision function more complex, and hence, as aforementioned, they can produce over-fitted models.

To measure the similarity of results between schemes, the Friedman test was applied (Demser, 2006). This is a non-parametric test used to detect significant differences in multiple classifiers. The obtained $p$-values for accuracy, g-means and sensibility are $0.1076,0.0150$ and 0.0083 , respectively. From these results, if an alpha level is fixed to $5 \%$, it can be concluded the following:

- For the accuracy measure, as long as the $p$-value in the Friedman test is 0.1076 , it can be concluded that there is no significant evidence of difference for the three methods.

- With respect to the g-mean metric, as the $p$-value is 0.0150 , the Friedman test detected significant differences. Furthermore, the test indicates that there is not significant difference between post-processing strategy and cost-sensitive SVM methods. Nevertheless, there is evidence that these classifiers and SMOTE are significantly different.

- With respect to the sensitivity measure, the $p$-value is 0.0083 , that is, the Friedman test detected significant differences. Furthermore, the test indicates that there is significant difference among the postprocessing strategy and the other two methods. Therefore, it can be concluded that the post-processing strategy is the best strategy to maximize the sensitivity metrics.

\section{Conclusions and Future Work}

From the experimental results on datasets with different degrees of imbalance, we can conclude that SVM performance is significantly improved using a new bias that considers the proportion of classes. An im- 
Table 3. Comparison of the novel method vs the cost-sensitivity and SMOTE approaches. Average values for accuracy, g-means and sensitivity for each dataset, using ten-fold crossvalidation.

\begin{tabular}{|c|c|c|c|c|c|c|c|c|c|}
\hline \multirow[t]{2}{*}{ Datasets } & \multicolumn{4}{|c|}{ accuracy } & \multicolumn{3}{|c|}{ g-means } & \multicolumn{2}{|c|}{ sensitivity } \\
\hline & $b_{p}$ & cost & SMOT1 & $b_{p}$ & cost & SMOTE & $\mathrm{E} b_{p}$ & cost & $\overline{\text { SMOTE }}$ \\
\hline Wineq_white (3) & .968 & .950 & .963 & .449 & .147 & .497 & .235 & .050 & .328 \\
\hline Abalone (19) & .491 & .739 & .584 & .625 & .682 & .605 & .828 & .648 & .646 \\
\hline Wineq_red (8) & .506 & .787 & .789 & .603 & .653 & .588 & .835 & .650 & .458 \\
\hline Page-Blocks (5) & .761 & .932 & .808 & .858 & .795 & .704 & .974 & 690 & .619 \\
\hline Yeast (7) & .649 & .896 & .902 & .749 & .875 & .876 & .891 & .860 & .853 \\
\hline Thyroid (1) & .928 & .975 & .989 & .950 & .957 & .885 & .976 & .934 & .795 \\
\hline Nursey (3) & .982 & .982 & .955 & .983 & .991 & .977 & .986 & 1.00 & 1.00 \\
\hline Fault (5) & .938 & .945 & .697 & .594 & .353 & .627 & .417 & .178 & .579 \\
\hline Wineq_white (4) & .764 & .839 & .897 & .621 & .657 & .627 & .513 & .515 & .441 \\
\hline Yeast (5) & .464 & .867 & .863 & .628 & .843 & .871 & .911 & .834 & .775 \\
\hline Muns (3) & .953 & .962 & .964 & .903 & .951 & .981 & .858 & .939 & .998 \\
\hline Letter (a) & .994 & .997 & .998 & .995 & .997 & .995 & .996 & .995 & .991 \\
\hline Car (3) & .973 & .899 & .903 & .941 & .946 & .948 & .911 & 1.00 & 1.00 \\
\hline Derma (2) & .951 & .931 & .904 & .944 & .935 & .917 & .936 & .944 & .939 \\
\hline Ecoli (5) & .968 & .943 & .945 & .950 & .976 & .959 & .945 & 1.00 & .978 \\
\hline Balance(2) & .774 & .584 & .813 & .830 & .781 & .801 & .909 & .684 & .796 \\
\hline GTC & .882 & .972 & .887 & .920 & .941 & .894 & .970 & .907 & .904 \\
\hline User modeling (1) & .981 & .950 & .949 & .968 & .933 & .926 & .956 & .961 & .904 \\
\hline Sat (4) & .868 & .919 & .921 & .894 & .892 & .863 & .929 & .861 & .799 \\
\hline Satimage (4) & .911 & .934 & .919 & .889 & .847 & .863 & .865 & .749 & .803 \\
\hline Euthyroid & .761 & .897 & .887 & .809 & .826 & .883 & .887 & .782 & .881 \\
\hline Glass (7) & .879 & .896 & .945 & .898 & .905 & .897 & .935 & .863 & .842 \\
\hline Segment (1) & .993 & .995 & .996 & .994 & .994 & .991 & .995 & .992 & .985 \\
\hline Hepatitis & .721 & .817 & .695 & .732 & .740 & .589 & .855 & .720 & .440 \\
\hline Column & .872 & .864 & .866 & .873 & .874 & .887 & .883 & .898 & .926 \\
\hline $\mathrm{Cmc}(2)$ & .630 & .668 & .737 & .618 & .643 & .595 & .607 & .556 & .435 \\
\hline Dna & .949 & .971 & .965 & .957 & .959 & .955 & .972 & .941 & .937 \\
\hline Vehicle (1) & .983 & .983 & .964 & .985 & .983 & .972 & .988 & .985 & .988 \\
\hline Transfusion & .748 & .698 & .522 & .614 & .633 & .548 & .462 & .546 & .615 \\
\hline Haberman & .634 & .721 & .642 & .617 & .485 & .572 & .606 & .359 & .490 \\
\hline German & .623 & .728 & .714 & .660 & .691 & .657 & .804 & .629 & .557 \\
\hline Waveform (0) & .873 & .884 & .853 & .884 & .888 & .856 & .921 & .901 & .866 \\
\hline Pima Diabetes & .742 & .733 & .634 & .725 & .725 & 649 & .687 & .694 & .723 \\
\hline TicTac (2) & .997 & .983 & .984 & .996 & .983 & .982 & .995 & .987 & .978 \\
\hline
\end{tabular}

portant benefit of the proposed approach is that the standard optimization problem associated to the SVM is not modified.

Neither new parameters must be tuned, so the computational cost is practically insignificant. By comparing this strategy with the cost-sensitive and SMOTE approaches, the bias modification approach achieves superior performance in terms of sensitivity, and it does with a classification function far less complex in terms of number of support vectors. 
Table 4. Proportion of support vectors in relation with the number of training data.

\begin{tabular}{lrrr||lrrr}
\hline Datasets & \multicolumn{3}{c}{ number of support vectors } & Datasets & & \multicolumn{3}{c}{ number of support vectors } \\
\cline { 2 - 4 } \cline { 6 - 7 } & \multicolumn{1}{c}{$b_{p}$} & \multicolumn{1}{c}{ cost } & SMOTE & & $b_{p}$ & cost & SMOTE \\
\hline Wineq_white (3) & $\mathbf{1 . 7 9}$ & 7.93 & 16.52 & User modeling (1) & 12.02 & 17.83 & $\mathbf{6 . 7 8}$ \\
Abalone (19) & $\mathbf{6 . 0 8}$ & 55.99 & 52.78 & Sat (4) & $\mathbf{1 5 . 0 4}$ & 19.07 & 27.10 \\
Wineq_red (8) & $\mathbf{6 . 2 8}$ & 36.66 & 38.32 & Satimage (4) & $\mathbf{9 . 0 9}$ & 10.29 & 18.64 \\
Page-Blocks (5) & $\mathbf{8 . 3 1}$ & 18.19 & 59.13 & Euthyroid & $\mathbf{2 2 . 0 0}$ & 38.00 & 31.25 \\
Yeast (7) & $\mathbf{4 . 1 8}$ & 27.91 & 28.79 & Glass (7) & 17.76 & 19.16 & $\mathbf{1 3 . 5 5}$ \\
Thyroid (1) & $\mathbf{2 . 4 9}$ & 11.24 & 9.92 & Segment (1) & $\mathbf{4 . 0 7}$ & 4.89 & 48.72 \\
Nursey (3) & $\mathbf{3 . 3 6}$ & 5.14 & 11.39 & Hepatitis & $\mathbf{3 2 . 5 6}$ & 47.29 & 38.37 \\
Fault (5) & $\mathbf{6 . 0 8}$ & 6.65 & 54.53 & Column & $\mathbf{2 5 . 8 0}$ & 26.77 & 29.51 \\
Wineq_white (4) & $\mathbf{1 1 . 4 7}$ & 30.26 & 31.37 & Cmc(2) & 44.26 & 64.09 & $\mathbf{4 1 . 9 8}$ \\
Yeast (5) & $\mathbf{7 . 3 5}$ & 55.02 & 28.66 & Dna & 37.35 & 39.85 & $\mathbf{1 8 . 0 5}$ \\
Muns (3) & 5.87 & 52.90 & $\mathbf{5 . 8 5}$ & Vehicle (1) & 9.69 & $\mathbf{9 . 3 4}$ & 22.87 \\
Letter (a) & 1.36 & 2.18 & $\mathbf{1 . 2 7}$ & Transfusion & $\mathbf{4 2 . 6 5}$ & 57.62 & 58.82 \\
Car (3) & $\mathbf{8 . 2 2}$ & 17.48 & 17.53 & Haberman & 52.28 & 65.03 & $\mathbf{3 8 . 2 3}$ \\
Derma (2) & $\mathbf{1 4 . 8 0}$ & 25.42 & 30.31 & German & $\mathbf{4 5 . 7 0}$ & 50.60 & 36.25 \\
Ecoli (5) & $\mathbf{7 . 7 4}$ & 31.25 & 19.05 & Waveform (0) & $\mathbf{2 2 . 8 4}$ & 24.12 & 43.88 \\
Balance (2) & $\mathbf{1 7 . 4 4}$ & 53.92 & 21.28 & Pima Diabetes & $\mathbf{4 8 . 9 5}$ & 52.60 & 55.86 \\
GTC & $\mathbf{7 . 3 8}$ & 8.98 & 67.38 & TicTac (2) & 35.59 & 36.01 & $\mathbf{1 8 . 4 7}$ \\
\hline
\end{tabular}

As future work, a theoretical framework for studying the movements of the bias in the workspace according to their definition is being developed.

\section{References}

AKBANI, R., KWEK, S., and JAPKOWICZ, N. (2004), "Applying Support Vector Machines to Imbalanced Datasets", in Proceedings of 15th European Conference on Machine Learning ECML'2004, pp. 39-50.

BATUWITA, R., and PALADE, V. (2010), "FSVM-CIL: Fuzzy Support Vector Machines for Class Imbalance Learning", IEEE Transactions on Fuzzy Systems, 18, 558-571.

BATUWITA, R., and PALADE, V. (2013), "Class Imbalance Learning Methods for Support Vector Machines", in Imbalanced Learning: Foundations, Algorithms, and Applications, pp. 83-99, Berlin, Germany: John Wiley and Sons.

CASTRO, C.L., CARVALHO, M.A., and BRAGA, A.P. (2009), "An Improved Algorithm for SVMs Classification of Imbalanced Data Sets", in Proceedings of 11th International Conference on Enginnering Applications of Neural Networks EANN 2009, pp. 108118.

CHAWLA, N.V., BOWYER, K.W., HALL, L.O., and KEGELMEYER, W.P. (2002), "SMOTE: Synthetic Minority Over-Sampling Technique", Journal of Artificial Intelligence Research, 16, 321-357.

COHEN, G., HILARIO, M., SAX, H., HUGONNET, S., and GEISSBUHLER, A. (2006), "Learning from Imbalanced Data in Surveillance of Nosocomial Infection", Artificial Intelligence in Medicine, 37, 7-18.

CRISTIANINI, N., and SHAWE-TAYLOR, J. (2000), An Introduction to Support Vector Machines and Other Kernel-based Learning Methods (1st ed.), New York, NY: Cambridge University Press. 
DEMSER, J. (2006), "Statistical Comparisons of Classifiers over Multiple Data Sets, Journal of Machine Learning Research, 7, 1-30.

ERTEKIN, S. (2013), “Adaptive Oversampling for Imbalanced Data Classification”, in Information Sciences and Systems, Lecture Notes in Electrical Engineering, 264, 261-269.

FRANK, A., and ASUNCION, A. (2010), UCI "Machine Learning Repository", University of California, School of Information and Computer Science, Irvine, http://archive.ics. uci.edu/ml.

GONZALEZ-ABRIL, L., NÚÑEZ, H., ANGULO, C., and VELASCO, F. (2014), "GSVM: An SVM for Handling Imbalanced Accuracy Between Classes in Bi-Classification Problems", Applied Soft Computing, 17, 23-31.

GONZALEZ-ABRIL, L., ANGULO, C., VELASCO, F., and ORTEGA, J.A. (2008), “A Note on the Bias in SVMs for Multiclassification", IEEE Transactions on Neural Networks, 19(4), 723-725.

HE, H., and GARCIA, E.A. (2009), "Learning from Imbalanced Data", IEEE Transactions on Knowledge and Data Engineering, 21(9), 1263-1284.

HE, H., and GHODSI, A. (2010), "Rare Class Classification by Support Vector Machine", in Proceedings 20th International Conference on Pattern Recognition, ICPR'10, pp. 548-551.

HERNÁNDEZ-SANTIAGO, J., CERVANTES, J., CHAU, A.L., and GARCÍA-LAMONT, F. (2012), "Enhancing the Performance of SVM on Skewed Data Sets by Exciting Support Vectors", in Proceedings of 13th Ibero-American Conference on Artificial Intelligence IBERAMIA 2012, pp. 101-110.

IMAM, T., TING, K.M., and KAMRUZZAMAN, J. (2006), "z-SVM: An SVM for Improved Classification of Imbalanced Data", in Proceedings of 19th Australian Conference on Artificial Intelligence AUS-AI 2006, pp. 264-273.

KANG, P., and CHO, S. (2006), "EUS SVMs: Ensemble of Under-Sampled SVMs for Data Imbalance Problems", Lecture Notes in Computer Science, 4232, 837-846.

LI, B., HU, J., and HIRASAWA, K. (2008), “An Improved Support Vector Machine with Soft Decision-Making Boundary", in Proceedings of 26th IASTED International Conference on Artificial Intelligence and Applications AIA'08, pp. 40-45.

LI, P., YU, X., BI, T.T., and HUANG, J.L. (2014), "Imbalanced Data SVM Classification Method Based on Cluster Boundary Sampling and DT-KNN Pruning", International Journal of Signal Processing, Image Processing and Pattern Recognition, 7(2), 6168.

LIU, Y., AN, A., and HUANG, X. (2006), "Boosting Prediction Accuracy on Imbalanced Datasets with SVM Ensembles", in Proceedings of 10th Pacific-Asia Conference on Knowledge Discovery and Data Mining PAKDD 2006, pp. 107-118.

LÓPEZ, V., FERNÁNDEZ, A., GARCÍA, S., PALADE, V., and HERRERA, F. (2013), "An Insight Into Classification with Imbalanced Data: Empirical Results and Current Trends on Using Data Intrinsic Characteristics", Information Sciences, 250, 113-141.

MUSCAT, R., MAHFOUF, M., ZUGHRAT, A., YANG, Y.Y., THORNTON, S., KHONDABI, A.V., and SORTANOS, S. (2014), "Hierarchical Fuzzy Support Vector Machine (SVM) for Rail Data Classification", in Proceedings of 19th IFAC World Congress, pp. 10652-10657.

NGUYEN, H.M., COOPER, E.W., and KAMEI, K. (2011), "Borderline Over-sampling for Imbalanced Data Classification", International Journal of Knowledge Engineering and Soft Data Paradigms, 3, 4-21. 
NÚÑEZ, H., GONZALEZ-ABRIL, L., and ANGULO, C. (2011), “A Post-Processing Strategy for SVM Learning from Unbalanced Data", in Proceedings 19th European Symposium on Artificial Neural Networks ESANN'2011, pp. 195-200.

ONETO, L., RIDELLA, S., and ANGUITA, D. (2016). “Tikhonov, Ivanov and Morozov Regularizationfor Support Vector Machine Learning, Machine Learning, 3, 103136.

RAMÍREZ, F., and ALLENDE, H. (2012), "Dual Support Vector Domain Description for Imbalanced Classification", in Artificial Neural Networks and Machine Learning ICANN 2012, Lecture Notes in Computer Science, 7552, 710-717.

SHANAHAN, J.G., and ROMA, N. (2003), "Improving SVM Text Classification Performance Through Threshold Adjustment", Lecture Notes in Computer Science, 2837, pp. 361-372.

SUKHANOV, S., MERENTITIS, A., DEBES, C., HAHN, J., and ZOUBIR, A. (2015), "Bootstrap-Based SVM Aggregation for Class Imbalance Problems", in Proceedings of 23rd European Signal Processing Conference EUSIPCO 2015, pp 155-169.

SUN, A., LIM, E.-P., and LIU, Y. (2009), "On Strategies for Imbalanced Text Classification Using SVM: A Comparative Study", Decision Support Systems, 48, 191-201.

SUN, Y., WONG, A.C., and KAMEL, M.S. (2009), "Classification of Imbalanced Data: A Review”, International Journal of Pattern Recognition and Artificial Intelligence, 23, 687-719.

TANG, Y., ZHANG, Y.-Q., CHAWLA, N.V., and KRASSER, S. (2009), "SVMs Modeling for Highly Imbalanced Classification", IEEE Transactions on Systems, Man and Cybernetics-Part B, 39, 281-288.

VAPNIK, V.N. (1999), The Nature of Statistical Learning Theory (Information Science and Statistics), New York, NY: Springer.

VEROPOULOS, K., CAMPBELL, C., and CRISTIANINI, N. (1999), "Controlling the Sensitivity of Support Vector Machines", in Proceedings of 16th International Joint Conference on Artificial Intelligence IJCAI 1999, pp. 55-60.

VILARIÑO, F., SPYRIDONOS, P., VITRIÀ, J., and RADEVA, P. (2005), "Experiments with SVM and Stratified Sampling with an Imbalanced Problem: Detection of Intestinal Contractions", in Proceedings of 3rd International Conference on Advanced Pattern Recognition ICAPR 2005, Vol. 2, pp. 783-791.

WANG, B.X., and JAPKOWICZ, N. (2010), "Boosting Support Vector Machines for Imbalanced Data Sets, Knowledge Information Systems, 25, 1-20.

WANG, H., and ZHENG, H. (2008), "An Improved Support Vector Machine for the Classification of Imbalanced Biological Datasets", in Proceedings of 4th International Conference on Intelligent Computation ICIC 2008, pp. 63-70.

WANG, H.-Y. (2008), "Combination Approach of SMOTE and Biased-SVM for Imbalanced Datasets", in Proceedings of International Joint Conference on Neural Networks IJCNN 2008, pp. 228-231.

WANG, Q. (2014), “A Hybrid Sampling SVM Approach to Imbalanced Data Classification", Abstract and Applied Analysis, Article ID 972786.

WASKE, B., BENEDIKTSSON, J.A., and SVEINSSON, J.R. (2009), "Classifying Remote Sensing Data with Support Vector Machines and Imbalanced Training Data", in Proceedings of 8th International Workshop on Multiple Classifier Systems MCS09, pp. 375-384. 
WU, G., and CHANG, E.Y. (2005), "KBA: Kernel Boundary Alignment Considering Imbalanced Data Distribution", IEEE Transactions on Knowledge and Data Engineering, 17, 786-795.

YANG, C.-Y., WANG, J., YANG, J.-S., and YU, G.-D. (2008), "Imbalanced SVM Learning with Margin Compensation", in Proceedings of 5th International Symposium on Neural Networks: Advances in Neural Networks ISNN'08, pp. 636-644.

YANG, P., ZHANG, Z., ZHOU, B.B., and ZOMAYA, A.Y. (2011), "Sample Subset Optimization for Classifying Imbalanced Biological Data", in Proceedings of 15th PacificAsia Conference on Advanced Knowledge Discovery and Data Mining PAKDD 2011, Vol. 2, pp. 333-344.

YU, T., DEBENHAM, J., JAN, T., and SIMOFF, S. (2006), "Combine Vector Quantization and Support Vector Machine for Imbalanced Datasets", in Artificial Intelligence in Theory and Practice, IFIP 19th World Computer Congress, Vol. 217, Chap. 9, pp. 81-88.

ZHOU, B., HA, M., and WANG, C. (2010), "An Improved Algorithm of Unbalanced Data SVM", Advances in Intelligent and Soft Computing, Fuzzy Information and Engineering, 78, pp. 549-555.

ZIȨBA, M., TOMCZAK, J.M., LUBICZ, M., and ŚWIA̧TEK, J. (2014), "Boosted SVM for Extracting Rules from Imbalanced Data in Application to Prediction of the Postoperative Life Expectancy in the Lung Cancer Patients", Applied Soft Computing, 14(Part A), 99-108. 